\title{
Structure and Oscillation of Underexpanded Jet
}

\author{
Hiromasa Suzuki ${ }^{1}$, Masaki Endo ${ }^{2}$, Yoko Sakakibara ${ }^{2}$ \\ ${ }^{1}$ Graduate School of Advanced Science and Technology, Tokyo Denki University, Saitama, Japan \\ ${ }^{2}$ Division of Electronic and Mechanical Engineering, Tokyo Denki University, Saitama, Japan \\ Email: 11udm01@ms.dendai.ac.jp
}

Received June 3, 2013; revised June 10, 2013; accepted June 17, 2013

Copyright (C) 2013 Hiromasa Suzuki et al. This is an open access article distributed under the Creative Commons Attribution License, which permits unrestricted use, distribution, and reproduction in any medium, provided the original work is properly cited.

\begin{abstract}
In this study, flow characteristics are experimentally and numerically discussed, especially concerning shock-cell structure and oscillatory phenomena of supersonic jet. The jet becomes underexpanded when the nozzle pressure ratio of convergent nozzle exceeds the critical value. The underexpanded jet is not uniform because of the presence of the expansion wave, the compression wave and the shock wave formed in it. Many vortices are induced in jet boundary region by shearing stress generated between supersonic jet flow and atmospheric air. They interact with shock waves composing cell node of jet, which is closely related to a noise radiating from the jet. In experiment, flow field is visualized and the shape of cell structure is examined. Furthermore, frequency of vortex generation is measured. The jet is simulated using TVD scheme and the results are compared with the experimental ones. Distortion of cell structure caused by three dimensional oscillation of jet and behavior of vortices are discussed.
\end{abstract}

Keywords: Underexpanded Jet; Plate Shock; Incident Shock; Visualization; TVD Scheme

\section{Introduction}

Underexpanded jet is one of the supersonic jets; when the nozzle pressure ratio of the convergent nozzle is higher than the critical value, the jet is underexpanded. In case of air, the critical pressure ratio is about 1.893. The expansion waves, which are generated at the nozzle lip, propagate in the jet and reflected at jet boundary as the compression waves. They converge to form a shock wave, and the expansion waves are generated again when the shock wave reaches the jet boundary. The jet is not uniform because these phenomena repeat and typical shock cell structure of the underexpanded jet is formed.

When the flat plate is placed perpendicularly to the jet, the strong wave called "plate shock" appears in front of the plate. The air passes through the plate shock, and its velocity becomes subsonic. The flow changes its direction and the wall jet is formed along the plate.

The structure of underexpanded impinging jet is shown in Figure 1. Such a flow field is often observed in the assist gas of laser cutting [1], the cooling jet of glass tempering process $[2,3]$ and the exhaust jet of VSTOL aircraft or rocket. The flow field causes several issues, for example, behavior of jet disturbed by vortex of the jet boundary and high frequency noise generation called "screech tone" [4-7].

In this study, shadowgraph method and Schlieren method were used to visualize the flow field. Furthermore, a period of generation cycle of vortex near the jet boundary was measured using a device comprising a laser and a photoelectric sensor.

The behavior of jet and vortices near the jet boundary were examined. In addition, under the same conditions, the numerical study was carried out using TVD scheme.

\section{Experimental Apparatus and Numerical Technique}

\subsection{Experimental Considerations}

The piping system of the experimental apparatus is shown in Figure 2. The air is compressed by screw

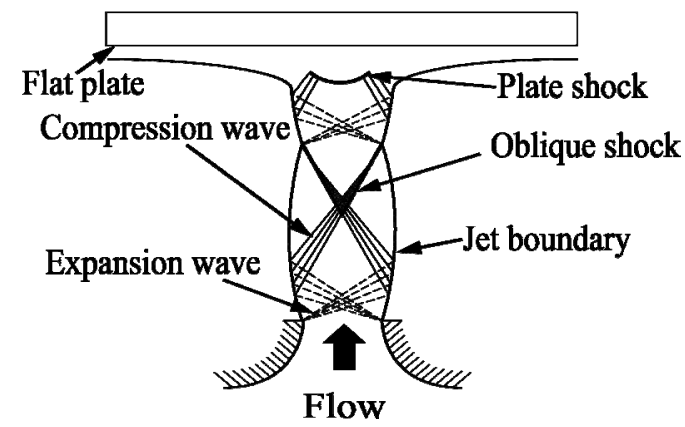

Figure 1. Schematic of underexpanded impinging jet. 


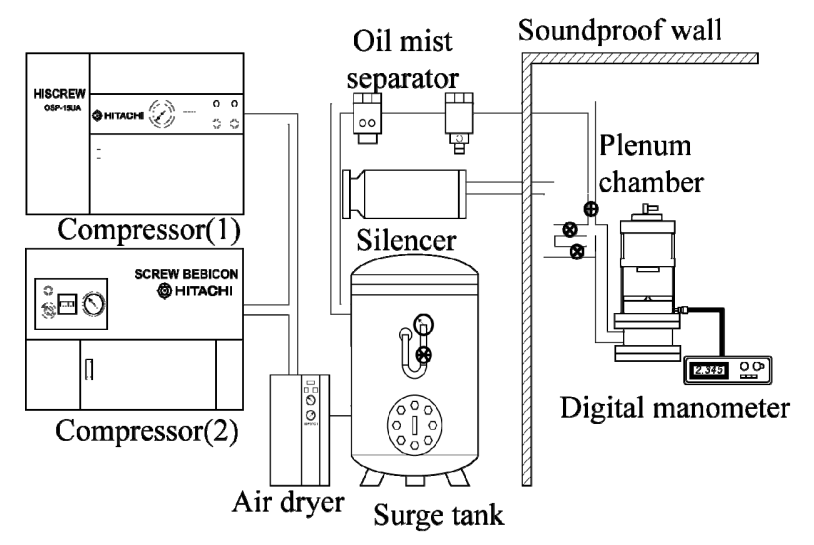

Figure 2. Piping system.

compressors and passes through the air dryer, surge tank, and oil mist separator. The compressed air is supplied into the plenum chamber to which convergent nozzle is attached in the soundproof room. The stagnation pressure of the air in the plenum chamber can be manually regulated by two gate valves and is measured by a digital manometer. An acoustic absorbent sheet is glued to the exit plane of the convergent nozzle. In this study, the convergent nozzle is adopted. The diameter of the convergent nozzle exit is $D=10 \mathrm{~mm}$ and the radius of the internal surface of the nozzle $R=20 \mathrm{~mm}$, which is well finished. And a flat plate is placed to be perpendicular to the jet axis, downstream of the nozzle.

The experiment is carried out under the condition of the nozzle pressure ratio $p_{0} / p_{a}=3.0$ to 3.8 with 0.1 steps and the nozzle plate distance $l / D=1.5$ to 2.5 with 0.1 steps, where $p_{0}$ is the pressure in the plenum chamber and $p_{a}$ the atmospheric pressure. The behavior of jet, vortex moving along jet boundary and plate shock above plate was examined under the conditions as mentioned above.

The flow fields were visualized using Schlieren photography and shadowgraphy. The spark bulb is employed as source of light, which emits flushing light duration a period of 180 ns.

Furthermore, the advective motion of vortex near the jet boundary is measured using a device composed of a laser and a photoelectric sensor, i.e. a simple Schlieren system. A semi-conductor type laser is used the beam width being $l_{w}=3 \mathrm{~mm}$. The vortex measurement system is shown in Figure 3. The knife edge is installed to cause it to block out the light. Going through the vortex, the laser beam is refracted in a direction away from the vortex core, and then, the illumination of beam reachable the sensor increases. Thus, the sensor detects the moving vortex. The laser is set at the position of antinode of second cell where the vortex starts to be organized. The antinode position is measured by means of the Schlieren pictures.

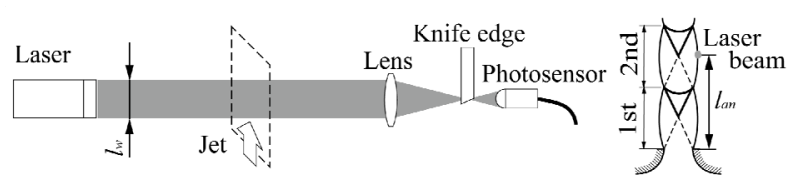

Figure 3. Vortex measurement system.

\subsection{Numerical Scheme}

The underexpanded jet impinging on the plate without an elastic deformation is numerically simulated. The computational flow field is assumed to be axisymmetric and Euler equations are employed as the governing equations. They are solved using 2nd-order TVD scheme proposed by Yee [8], with minmod function as a stable limiter function [9].

The computational region used in this study is covered with a structured mesh. The radial distance of the region is 7.5 diameters of the converging nozzle, where 150 computational grids are clustered towards the jet axis. Along the jet axis there are 100 grids at uniform intervals. The critical condition estimated from the stagnant properties in the plenum chamber is specified at the exit plane of the nozzle. The boundary condition is given to be reflective at the jet axis. The nozzle wall and the plate are slip wall boundary and, at the other boundaries, the Riemann variables are kept at constant.

\section{Results and Discussion}

\subsection{Behavior of Free Jet}

Figure 4 shows the flow field visualized using Schlieren method. These pictures were taken at random under the same condition. In these figures, the underexpanded free jet issues from the nozzle in the lower side of the picture at the pressure ratio $p_{0} / p_{a}=3.5$. The knife edge is placed in the direction normal to jet axis so that the expansive region is dark and compressive region bright. As can be seen in these pictures, the dark region and the bright region are deformed and the deformation becomes stronger as further away from the nozzle. Thus, the jet is found to be unsteady and oscillate laterally or helically.

In order to quantitatively evaluate the unsteady behavior of the jet, the statistical analysis is conducted through the visualization of the flow field. 50 pictures were taken at random at each pressure ratio, and the instantaneous shape of the jet was measured, e.g. the cell length $l_{c}$ and the inclination of cell node $\alpha_{n}$ as shown in Figure 5. The cell node between the 1st and 2nd cell, i.e. end node of cell, is defined as 1st cell node, and so on.

\subsection{Cell Length}

Figure 6 shows deviations of cell length of 1st, 2nd and 3rd cell at each pressure ratio. The empty plots show the 


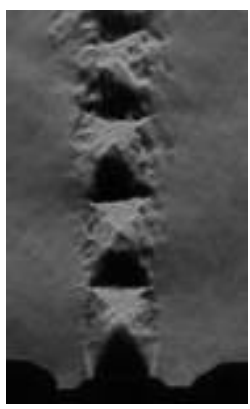

(a)

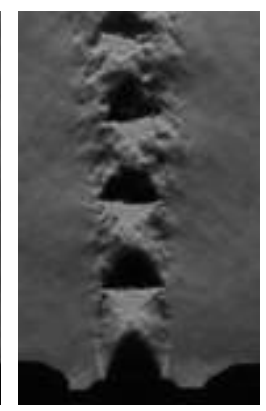

(b)

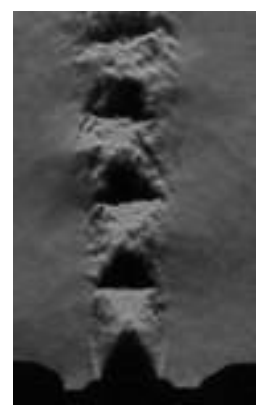

(c)

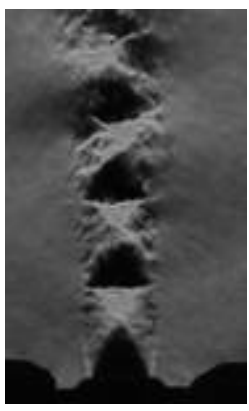

(d)

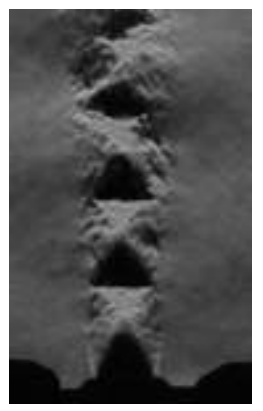

(e)
Figure 4. Schlieren photograph $\left(p_{0} / p_{\mathrm{a}}=3.5\right)$.

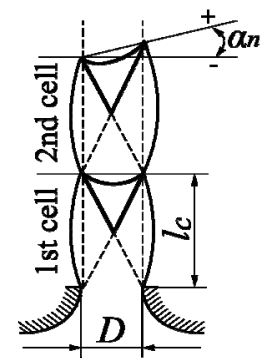

Figure 5. Measurement points.

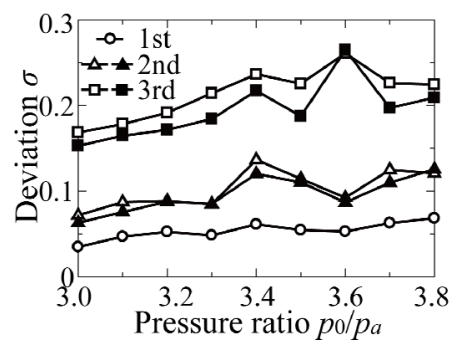

Figure 6. Deviation of cell length.

lengths of individual cell, and the solid ones total lengths between the nozzle exit and the end node of corresponding cell. Overall, these plots gradually rise as the pressure ratio increases and these take extreme values at $p_{0} / p_{\mathrm{a}}=$ 3.4 and 3.6. Concerning the individual cell length, the deviation for downstream cell is seen to swell two-fold. Thus, the amplitude of the oscillation in length becomes larger downstream.

Comparing the deviations between the individual and the total lengths, the deviation of total length is smaller than that of individual length regardless of the pressure ratio. This means that the 3rd cell shrinks if the 2nd cell stretches, that is, the changes of length caused by oscillation of the neighboring cells are cancelled as a whole. Observing the difference between the deviations at each pressure ratio carefully, the oscillation at $p_{0} / p_{\mathrm{a}}=3.6$ may have the same phase.

\subsection{Cell Node Angle}

Figure 7 shows deviation of cell node angles $\alpha_{n}$ of 1 st, 2nd and 3rd cell. This deviation is related to three-dimensional oscillation because $\alpha_{n}$ means the angle of node against jet axis as shown in Figure 5. The value of the deviation almost linearly rises with the increase in the pressure ratio and becomes larger downstream, e.g., $\sigma_{1}=$ $0.9, \sigma_{2}=2.2$ and $\sigma_{3}=4.3$ at $p_{0} / p_{\mathrm{a}}=3.4$. Hence, this means that the hydrodynamic instability along the jet boundary grows downstream.

\subsection{Behavior of Impinging Jet}

The experiment is carried out under the same condition of $p_{0} / p_{\mathrm{a}}$ as the free jet and the pictures are statistically analyzed. 50 pictures were taken at random in each nozzle plate distance. The geometric characteristics of the jet structure were measured, e.g. the positions of vertex and base of the plate shock $\left(l_{v p}\right.$ and $\left.l_{b p}\right)$, the plate shock diameter $d_{p}$ and the inclination of plate shock $\alpha_{p}$ as shown in Figure 8.

Figure 9 shows the deviation against the pressure ratio. The deviation $\sigma$ in the ordinate is normalized by the deviation $\sigma_{s}$ at $l / D=1.5$. It can be seen that the deviation of $\alpha_{p}$ undergoes abrupt change from $l / D=1.6$ to 1.8 and that the fluctuation of $l_{v p}$ stands out at $l / D=1.8$. These comparatively high values represent that the jet strongly oscillates under this condition. Especially at $l / D=1.8$, deviation of $l_{v p}$ stands out and this means that the center part of plate shock strongly oscillates in axially-symmetric pattern.

Furthermore, the deviation of $\alpha_{p}$ also has high value at $l / D=1.7$, and this means that lateral or helical motion occurs simultaneously. Thus, the plate shock intensely

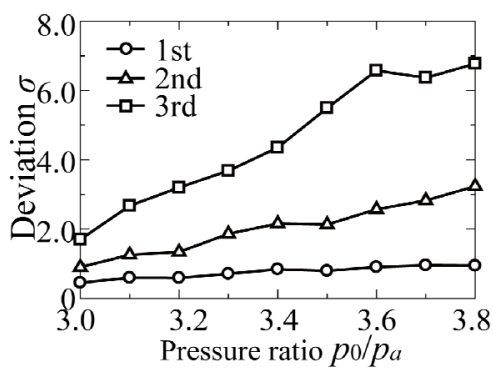

Figure 7. Deviation of angle of cell node. 


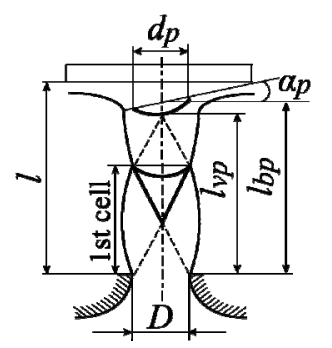

Figure 8. Measurement points.

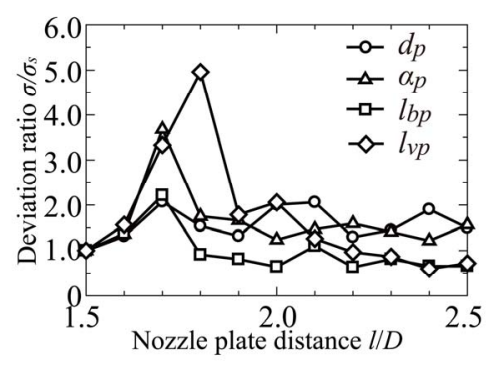

Figure 9. Deviation of plate shock.

oscillates at a certain nozzle plate distance.

Figure 10 shows averages of $l_{v p}$ and $l_{b p}$ at $p_{0} / p_{\mathrm{a}}=3.4$. The dashed line shows the position of 1st cell node obtained from pictures of free jet. Position of plate shock moves rapidly downstream to approach the plate at between $l / D=1.6$ and $l / D=1.7$. Distance between the vertex and base of the plate shock increase in the range from $l / D=1.7$ to $l / D=1.8$. These phenomena seem to be affected by location of the vertex of plate shock; whether the vertex is in the compression region or not. In the compression region, the plate shock crosses the incident shocks from the nozzle lip before they reach the jet axis, while in the expansion region, the incident shocks intersect each other on jet axis as shown in the next.

Figure 11 shows shadowgraph pictures of impinging jet at various nozzle plate distances from $l / D=1.5$ to 2.5. The jet issues from the nozzle exit on the bottom of each picture. Plate shock crosses the incident shocks at $l / D=$ 1.5 and 1.6, and at $l / D>1.7$, the intersection of incident shock appears upstream of plate shock. In addition, a weak shock wave can be seen in the region between the plate shock and the plate. This weak shock appears up to $l / D=1.6$ and disappears at $l / D=1.7$. At $l / D>1.8$, the plate shock is in the expansion region of second cell.

Details of shock structure can be seen in the results obtained by the numerical simulation. Figures 12 show the density contours (left-side half of each figure) and the pressure contours (right-side half of each figure) at different nozzle plate distance between $l / D=1.4$ and 2.0. The flow in the region between the plate shock and the plate is seen to be complex with $l / D$ smaller than $l / D=$ 1.6. Under such conditions, because the intersection of the incident shocks in the jet is located downstream of

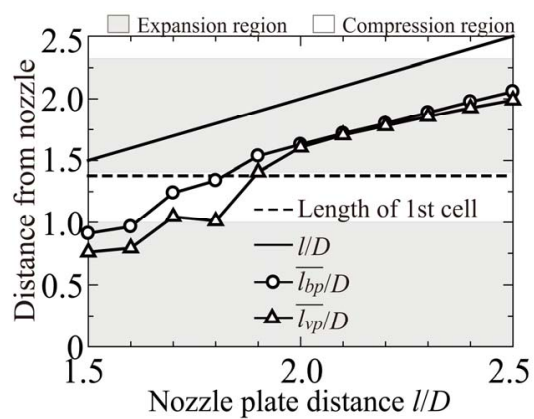

Figure 10. Distance of plate shock from nozzle.

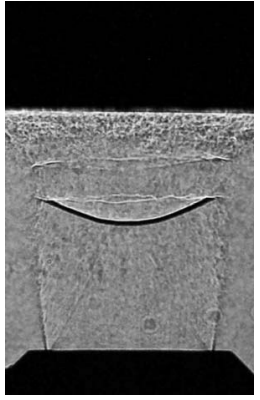

(a)

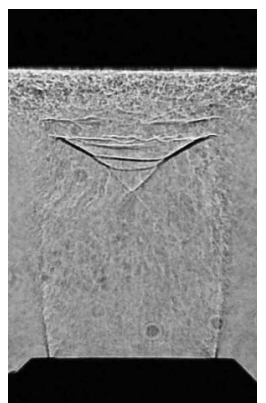

(d)

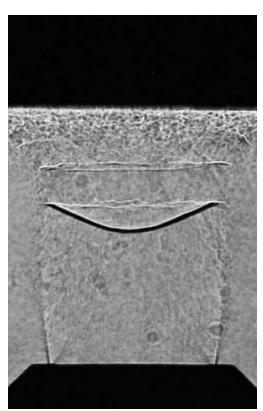

(b)

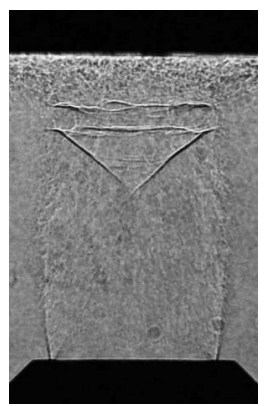

(e)

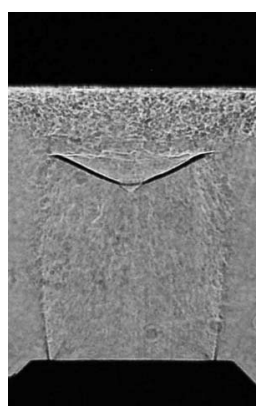

(c)

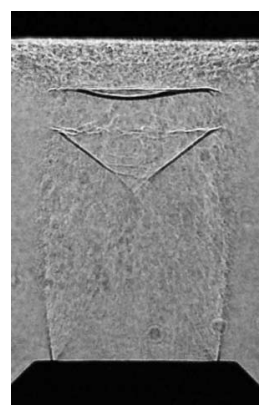

(f)
Figure 11. Shadowgraph photograph $\left(p_{0} / p_{\mathrm{a}}=3.4\right)$. (a) $I / D=$ 1.5; (b) $l / D=1.6$; (c) $l / D=1.7$; (d) $l / D=1.8$; (e) $l / D=1.9$; (f) $l / D=2.0$.

the plate shock, the strength of plate shock near the jet axis is stronger than that in the surrounding region. Therefore, contact surface is generated downstream of the plate shock and the iteration of reflection of waves between jet boundary and the contact surface occurs. Weak shock wave seen in Figures 11(a) and (b) is considered to be the shock wave which consists of converged compression wave during this iteration. The weak shock wave disappears at $l / D=1.7$, where the intersection of the incident shocks is upstream of plate shock (see Figure 11(c) and Figure 12(e)). In Figure 12(f), plate shock forms close to the incident shock and they are partly piled up. This can be seen also in Figure 11(d).

The position of plate shock changes into second cell at larger $l / D$. 


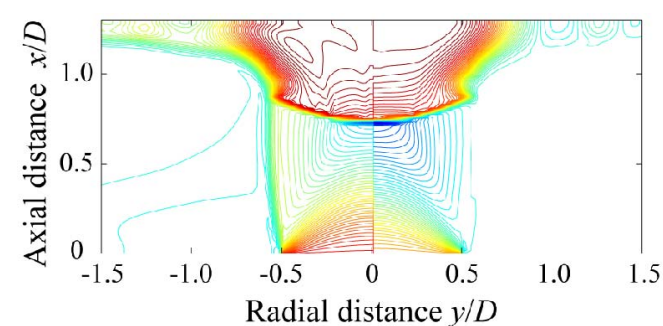

(a)

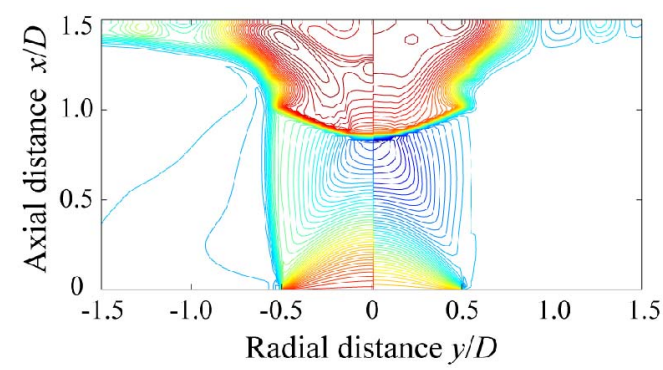

(c)

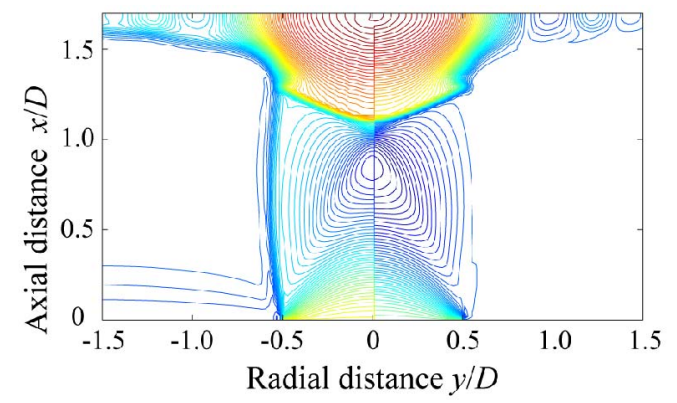

(e)

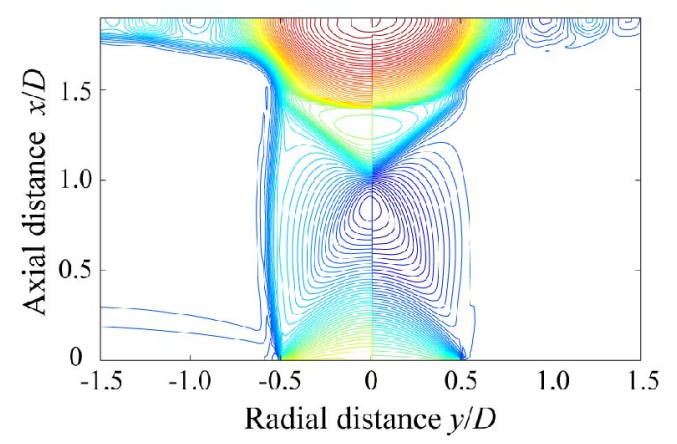

(g)

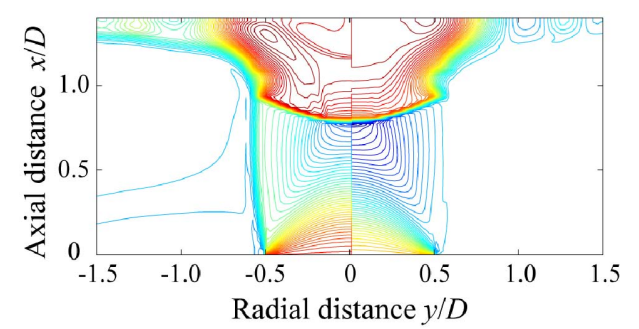

(b)

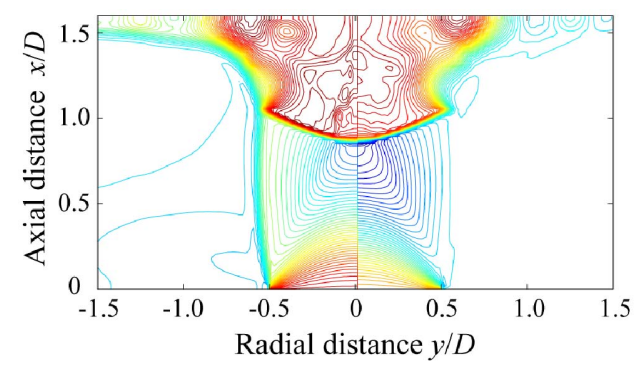

(d)

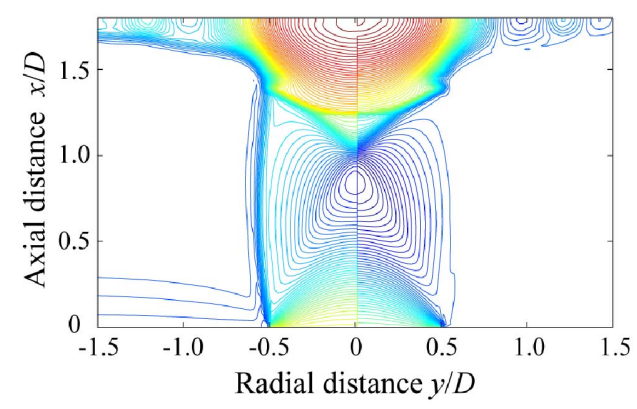

(f)

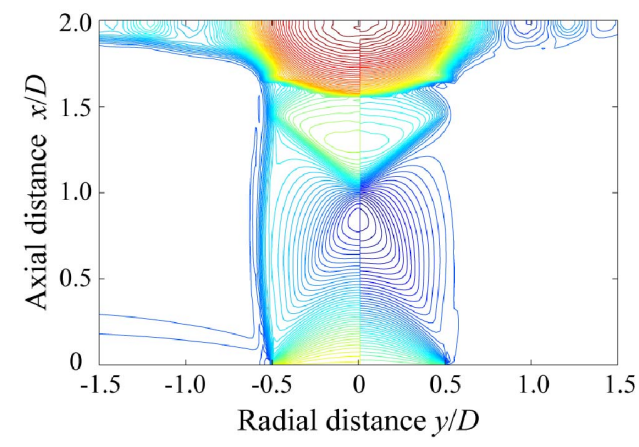

(h)

Figure 12. Numerical result $\left(p_{0} / p_{\mathrm{a}}=3.4\right.$ ): (a) $l / D=1.3$; (b) $l / D=1.4$; (c) $l / D=1.5$; (d) $l / D=1.6$; (e) $l / D=1.7$; (f) $l / D=1.8$; (g) $l / D=1.9 ;$ (h) $l / D=2.0$.

\subsection{Frequency of the Vortex Near the Free Jet Boundary}

Figure 13 shows the output signal history measured by the photoelectric sensor at $p_{0} / p_{\mathrm{a}}=3.0$. The sharp drop of the voltage means that a vortex passed through a laser. The vortices are seen to intermittently go through the measuring point. To search the periodicity of the vortex movement, the output signal is analyzed using FFT. The change of the dominant frequencies against the pressure rise is shown in Figure 14. The empty plot denotes the dominant frequency obtained though the FFT analysis of output data as in Figure $\mathbf{1 3}$ at each pressure ratio. The solid plots are obtained from the experimental results of the sound pressure wave, which comes from the paper by S. Tamura and J. Iwamoto [10]. The sound pressure wave has two components of the frequencies. Those dominant frequencies lie around $15 \mathrm{kHz}$ and $25 \mathrm{kHz}$, 


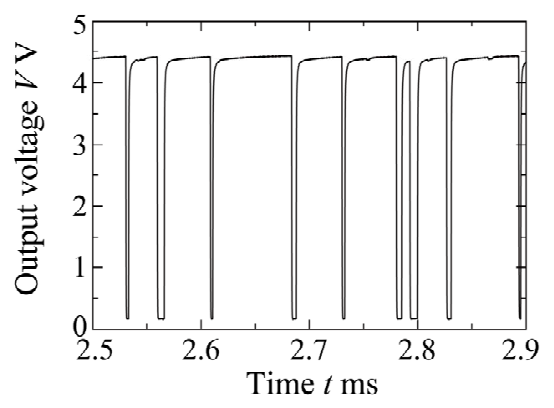

Figure 13. Output signal wave $\left(p_{0} / p_{a}=3.0\right)$.

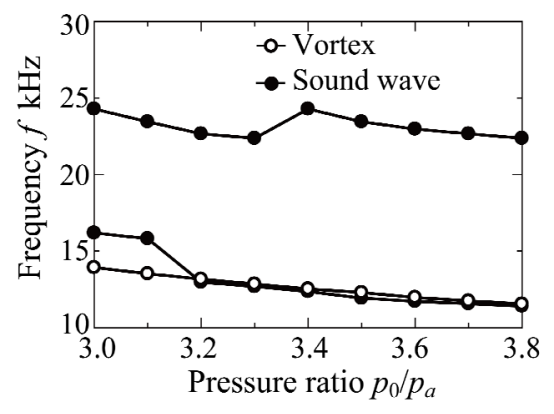

Figure 14. Frequency of vortex generation and sound wave.

slightly decreasing with the rise of pressure ratio. The higher frequency jumps at $p_{0} / p_{\mathrm{a}}=3.3$ and lower one drops at 3.1. These discontinuous changes are known to be caused by the structure of the underexpanded jet. As can be seen in Figure 14, the change of lower frequency quantitatively agrees well with that of the vortex. It is evident that the sound emitted form the jet is cause by the behavior of the vortex moving along the jet boundary.

\section{Conclusions}

To investigate the oscillatory phenomena of underexpanded jet, the flow field was visualized using the schlieren photography, and shadowgraphy and the behavior of vortex near the jet boundary was observed. As a result, the following conclusions were drawn:

- The deviation of cell node angle linearly increases with rise of pressure ratio.

- The deviation of cell length takes maxima and min- ima at some pressure ratios.

- The weak shock is formed in the downstream region of plate shock, when the vertex of plate shock locates near the end of expansion region.

- Good agreement is found between frequency of vortex generation and that of sound wave.

\section{REFERENCES}

[1] J. Fieret, M. J. Terry and B. A. Ward, "Overview of Flow Dynamics in Gas-Assisted Laser Cutting," SPIE High Power Lasers, Vol. 801, 1987, pp. 243-250.

[2] S. Aratani and N. Ojima, "Effect of Shock Waves on Fracture and Quenching in Tempered Glass,” Proceedings of the 18th International Symposium of Shock Waves, Sendai, 21-26 July 1991, pp. 1277-1282.

[3] J. M. Barsom, "Fracture of Tempered Glass," Journal of the American Ceramics Society, Vol. 51, No. 2, 1968, pp. 75-78. doi:10.1111/j.1151-2916.1968.tb11840.x

[4] A. Powell, Y. Umeda and R. Ishii, "Observation of the Oscillation Modes of Choked Circular Jets,” Journal of the Acoustical Society of America, Vol. 92, No. 5, 1992, pp. 2823-2839. doi:10.1121/1.404398

[5] A. Powell, "The Sound-Producing Oscillations of Round Underexpanded Jets Impinging on Normal Plates,” Journal of the Acoustical Society of America, Vol. 83, No. 2, 1988, pp. 515-533. doi:10.1121/1.396146

[6] C. K. W. Tam, "Broadband Shock Associated Noise from Supersonic Jets Measured by a Ground Observer,” AIAA Journal, Vol. 30, No. 10, 1992, pp. 2395-2401. doi:10.2514/3.11239

[7] J. Panda, "Shock Oscillation in Underexpanded Screeching Jets,” Journal of Fluid Mechanics, Vol. 363, 1998, pp. 173-198. doi:10.1017/S0022112098008842

[8] H. C. Yee, "Upwind and Symmetric Shock-Capturing Scheme,” National Aeronautics and Space Administration (NASA), Washington DC, 1987.

[9] Y. Sakakibara and J. Iwamoto, "Numerical Study of Oscillation Mechanism in Underexpanded Jet Impinging on Plate,” Journal of Fluids Engineering, Vol. 120, No. 3, 1998, pp. 477-481. doi:10.1115/1.2820687

[10] S. Tamura and J. Iwamoto, "Study on the Noise Generated from Underexpanded Free and Impinging Jet," Transactions of the Japan Society of Mechanical Engineers, Series B, Vol. 60, No. 579, 1994, pp. 283-289. 


\section{Nomenclature}

$D$ : Diameter of nozzle exit

$d_{p}$ : Diameter of plate shock

$f$ : Frequency

$l$ : Distance between nozzle exit and flat plate

$l_{c}$ : Length of cell

$l_{a n}$ : Position of antinode of second cell

$l_{b p}$ : Position of base of plate shock

$\bar{l}_{b p}$ : Average of position of base of plate shock

$l_{v p}$ : Position of vertex of plate shock

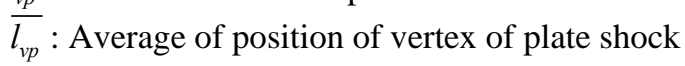

$l_{w}$ : Width of laser beam

$p_{a}$ : Atmospheric pressure

$p_{0}$ : Stagnation pressure in a plenum chamber

$R$ : Radius of convergent nozzle

$\alpha_{n}$ : Angle of cell node

$\alpha_{p}$ : Angle of plate shock

$\sigma$ : Deviation

$\sigma_{1}$ : Deviation of 1 st cell of free jet

$\sigma_{2}$ : Deviation of 2nd cell of free jet

$\sigma_{3}$ : Deviation of 3rd cell of free jet

$\sigma_{s}$ : Deviation at $l / D=1.5$ 